\title{
Multiobjective Optimization of Electrical Machine, a State of the Art Study
}

\author{
P.Ponmurugan \\ Assistant Professor, \\ Department of EEE, \\ EBET Group of Institutions, Kangayam.
}

\author{
N.Rengarajan, PhD. \\ Principal, \\ K.S.R College of Engineering, \\ Tiruchengode.
}

\begin{abstract}
This paper presents a literal study of Multiobjective optimization (MO) in general used in electrical machine optimization in the recent years. A set of a set of nonlinear constraints (modeling availability of resources) with a set of nonlinear objective functions (modeling several performance criteria) is solved with the help of Multi objective optimization (MO). The MO problem has several applications in science, engineering, finance, etc. It is normally not possible to find an optimal solution in $\mathrm{MO}$, since the various objective functions in the problem are usually in conflict with each other. Therefore, the objective in MO is to find the Pareto front of efficient solutions that provide a substitution between the various objectives. The paper will summon up some of the work done using Multiobjective optimization on electric machines in the last years. An overview of methods used will be given and the conclusion of the different papers will be presented.
\end{abstract}

\section{Keywords}

Multiobjective Optimization, Pareto front, Evolutionary, algorithms, induction machine.

\section{INTRODUCTION}

In recent years, optimization algorithms are focused by the research community as well as the industry. Scientifically, the field of optimization algorithms is a highly relevant research area, because these algorithms can find approximate solutions to NP-hard problems and solutions to problems where no analytic method exists, e.g. for solving non-linear differential equations.

Optimization process is used to find the solution where a set of objective functions is minimized or maximized with subject to different constraints. The optimization problem with multiple objectives is known as multi objective optimization (MO) problem. Multiobjective optimization [1-4] is also referred to as multi-criteria optimization, multi-performance or vector optimization [5].

An MO problem may be stated as finding a vector of decision variables that satisfies constraints and optimizes a vector function whose elements represent the objective functions [6]. A mathematical description of performance criteria obtained through the objective functions are usually in conflict with each other. Hence, the term optimization means finding a solution which would give the values of all the objective functions acceptable to the decision maker. Obtaining the optimal solution in a multi objective optimization problem is difficult one. This is because the multiple objective functions that are present often conflict each other and it is impossible to optimize all the objective functions at the same time. Instead, a set of solutions called best solutions providing a tradeoff between the objective functions can be found. The final solution for the specified application is selected from the set of best solutions by the problem solver.

One of the first applications of multi objective optimization was in economics to solve public investment problems in the 1960s [7]. Other applications arise in control [8], and water resource planning [9]. Multiobjective optimization is also frequently used in engineering, science, industry and finance $[1,2]$.

\section{MATHEMATICAL FORMULATION} OF A MULTIOBJECTIVE OPTIMIZATION PROBLEM

Consider the problem min $f(x)$ such that

$$
\begin{aligned}
& g_{j}(x) \leq 0, \quad j=1,2, \Lambda \Lambda, m \\
& h_{l}(x) \leq 0, \quad l=1,2, \Lambda \Lambda, p
\end{aligned}
$$

The vector $x \in R^{n}$ contains the decision variables in (1). The set

$S=\left\{x \in R^{n} \mid g_{j}(x) \leq 0, j=1,2, \Lambda \Lambda m, h_{l}(x)=0, l=1,2, \Lambda \Lambda p\right\}$ is the feasible region of (1) and depicts constraints such as the limited availability of resources in the problem. The mapping $f: R^{n} \rightarrow R^{k}$ defined by $f(x)=\left(f_{1}(x), f_{2}(x), \Lambda \Lambda f_{k}(x)\right)^{T}$ contains the $\mathrm{k}$ objective functions (possibly nonlinear) of (1). We define the feasible objective region $\mathrm{Z}$ as the image of the feasible region $\mathrm{S}$ under the mapping $f$, i.e., $Z=\left\{y \in R^{k} \mid y_{i}=f_{i}(x), i=1,2, \Lambda \Lambda, k, \forall x \in S\right\}$. It is assumed that the entire objective functions in $f(x)$ are being minimized. If an objective function $f_{i}(x)$ is to be maximized, it is equivalent to minimizing the function $f_{i}(x)$.

It is important to distinguish between the constraint space $S$ and the objective function space $\mathrm{Z}$ in multiobjective optimization. The set $\mathrm{Z}$ plays an important role in the concept of Pareto optimality in multiobjective optimization.

\section{METHODS \\ TO SOLVE MULTIOBJECTIVE OPTIMIZATION PROBLEMS}

The methods that are use to solve multiobjective optimization problems can be broadly classified as

1. Classical Methods

2. Evolutionary Algorithms. 


\subsection{Classical Methods}

In classical methods, the various objective functions of the multiobjective optimization problem are combined together in a single objective function.

$$
\hat{f}(x)=\sum_{i=1}^{k} w_{i} f_{i}(x)
$$

where $w_{i}>0$ are appropriate weights. The single objective function $\hat{f}(x)$ is minimized over the feasible set $\mathrm{S}$ using traditional nonlinear optimization techniques for a single objective function.

Classical methods are also classified as a priori or progressive techniques based on when the weights are assigned to the objective functions as follows:

\subsubsection{A priori}

In this method, the weights are assigned to the objective functions before optimization is performed. One of the requirements of this method is that the order of importance of the objectives has to be known ahead of time. Once the weights are chosen, they are fixed throughout the optimization procedure. Setting the weights before optimization omits desirable solutions from the model. For the assigned weights to be effective, the objective functions need to be normalized to factor in their different dynamic ranges. This is not an easy task because it requires the knowledge of the extreme values of the objective functions. These drawbacks make a priori preference methods [4] very difficult to use.

\subsubsection{Progressive}

In this method, the weights are updated periodically by the decision maker based on the current solution [4] in the optimization process. This method is better than the a priori technique because corrections are made using the information obtained during optimization. However, prior knowledge of the problem is often required to define a scheme of preference to bias the search so that the decision maker's biases do not lead to undesirable solutions.

To summarize, one of the main shortcoming of classical methods is that some prior knowledge of the problem is required to assign reasonable weights. In classical methods, it is not possible to find multiple solutions in a single run and also not possible to find all the Pareto optimal solutions. This causes the decision maker to miss out other desirable solutions to a problem. However, these algorithms are known to converge to a Pareto optimal solution of the multiobjective problem [10].

\subsection{Evolutionary Algorithms}

The classical methods are extensively being replaced by Evolutionary Algorithms (EA). Evolutionary algorithms are iterative and stochastic optimization techniques inspired by concepts from Darwinian evolution theory. An EA simulates an evolutionary process on a population of individuals with the purpose of evolving the best possible approximate solution to the optimization problem at hand.

Although, evolutionary strategies and genetic algorithms are categorized as evolutionary algorithms, they have an important difference: Evolutionary strategies encode parameters as floating point numbers and then manipulate those using arithmetic operators whereas genetic algorithms encode parameters as bit strings and then manipulate those using logical operators. So, evolutionary strategies are suitable for continuous optimization while genetic algorithms are more suitable in combinatorial optimization. Historically, EAs were first suggested in the 1940'ties [11]. However, the founding fathers of modern EAs, Evolutionary Programming [12], and Genetic Algorithms are considered. Several years later, Evolutionary Algorithms (EAs) and Evolutionary Computation (EC) were introduced as unifying terms for the forest of optimization techniques inspired by biological evolution. All evolutionary algorithms aim to improve the existing solution using the techniques of recombination, mutation, and selection. Fig. 1 illustrates the initialization and the iterative cycle in EAs.

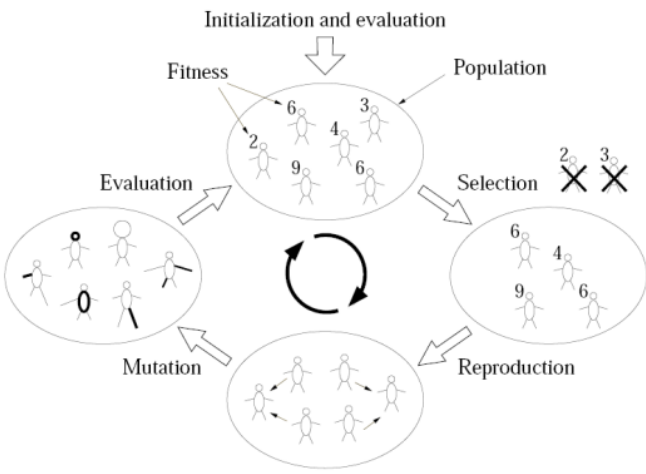

Fig 1: Initialization and the iterative cycle in evolutionary algorithms

The general paradigm is as follows:

\subsubsection{Initialization}

The initial population consisting of $\mu$ members (parents) is chosen randomly.

\subsubsection{Recombination}

The $\mu$ parent vectors randomly recombine with each other to produce $\lambda \geq \mu$ child vectors.

\subsubsection{Mutation}

After recombination, the $\lambda$ child vectors undergo mutation where a random deviation is added to each child vector. Selection: The two most commonly used strategies are $(\mu, \lambda)$ and $(\mu, \mu+\lambda)$ selection strategies. In $(\mu, \lambda)$ strategy, the best $\mu$ child vectors replace the existing $\mu$ parent vectors to become parents in the next generation, whereas in $(\mu, \mu+\lambda)$ strategy, the best $\mu$ vectors from the child and parent populations become parents in the next generation.

\subsubsection{Termination}

The number of iterations (generations) performed depends on the convergence criterion chosen.

A few advantages of evolutionary algorithms over classical methods:

Evolutionary algorithms are multiobjective optimization techniques that generate a set of equally desirable solutions using the concept of Pareto optimality. The decision maker chooses a solution from the set of available Pareto solutions, and thus implicitly assigns a set of weights. Unlike classical methods, no weights are assigned to the various objectives during the course of the algorithm. Therefore, the solutions are found without introducing bias.

In evolutionary algorithms, a population of desirable solutions is covenant in each iteration whereas in classical methods only one solution is focused. Unlike classical methods where 
a pre-defined rule is used to search through the solutions, evolutionary algorithms use probabilistic rules to search through solutions. Moreover, evolutionary algorithms are easier to implement and are typically faster than classical techniques. Moreover, several parallel implementations are currently available [13]

\section{MULTIOBJECTIVE OPTIMIZATION OF ELECTRICAL MACHINES: STUDY OF RECENT WORK}

\subsection{A multiobjective design optimization of induction machine using CAD and ANNs}

This paper [14] proposes a multiobjective optimization technique based on the Computer Aided Design (CAD) using finite element method in designing ventilation holes of squirrel cage induction motor and the Artificial Neural Network (ANN) optimization in the purpose of maximizing the ventilation effect without making the yoke-saturation more serious. Applying the traditional design methodology, the rotating machine model with the circle shaped ventilation holes is determined. By using the numerical analysis, the configurations of ventilation holes from the viewpoint of the magnetic saturation are improved. An optimal design of the holes, which maximizes the ventilation effect without making the rotor-yoke saturation serious, is performed. Finally, a novel optimization technique based on the Artificial Neural Network (ANN) enables to efficiently obtain the optimal solutions for practical induction electric machine design.

\subsection{Design optimization of electric motors} by multiobjective Fuzzy genetic algorithms

This paper [15] describes an approach to design the submersible induction motor with two objective functions: the full load torque and the manufacturing cost utilizing the concept of fuzzy sets, convex fuzzy decision-making and a genetic algorithm having feature of a unique search. In this method, the objective functions are combined by fuzzy memberships so that the chromosomes with best performances for all objective functions have more chances to be chosen for participation in the next generation. The multiobjective fuzzy genetic algorithm (MFGA) optimization results show the proposed approach is viable and reliable. The optimally designed motor is compared with an industrial motor having the same ratings. The results of optimal design show the reduction in the manufacturing cost and the improvement in the full load torque of the motor. The simulation results shown that the manufacturing cost decreased by $7 \%$ and the full load torque increased by $9 \%$.

\subsection{Multiobjective optimization of three- phase induction motor design based on genetic algorithm}

In this article [16], the multiobjective optimization technique based on the genetic algorithm and sizing equation is applied for the three phase squirrel-cage type induction motor. The optimization design is applied for the motor with 4 pole, $380 \mathrm{~V} / 60 \mathrm{~Hz}$ and $1.5 \mathrm{~kW}$. The objective functions are the decrease of the total electromagnetic losses and the increase of the locked torque respectively. The width of stator teeth, the depth of stator slot, the diameter of the winding coil and the depth of the rotor slot are also selected as the design variables. For the verification of the design result, the simulation and experimental data are compared. The design result was selected from Pareto optimal solution set. Compared to the initial model, the locked torque is increased by $16.4 \%$ and the efficiency of the motor is also improved $2.1 \%$ respectively.

\subsection{Multiobjective optimization design of in wheel switched reluctance motors in electric vehicles}

This paper [17] discusses three criterions the average torque, the average torque per copper loss, and the average torque per motor lamination volume to evaluate the design of SRMs in Electric Vehicle (EV) applications. These three criterions imply torque, efficiency and torque density respectively. To obtain high torque, low copper loss, and high torque density by using three weight factors and three base values, the authors developed the optimization function with multiobjectives, which is defined as the correct compromise between the three criterions. The optimized valuables are selected as the stator and rotor pole arc angles. The values of three criterions at different combinations of stator and rotor pole arc angles are compared. It can be seen that the optimal stator pole arc angle $\left(22^{\circ}\right)$ and the optimal rotor pole arc angle $\left(23^{\circ}\right)$ result in the best design. Using the data, the prototype of the four-phase in wheel SRM has been manufactured.

\subsection{Multiobjective parameter estimation of induction motor using Particle Swarm Optimization}

This paper [18] investigates the applicability of PSO algorithm for multi-objective parameter estimation to minimize the deviation between estimated and manufacturer data. Three different induction motor models such as approximate, exact and deep bar circuit models are estimated using PSO technique. The parameter estimation methodology describes a method for estimating the steady-state equivalent circuit parameters from the motor performance characteristics, which is normally available from the manufacturer data or from tests. The sensitivity analysis is performed to identify parameters, which have the most impact on motor performance. The feasibility of the proposed method is demonstrated for two different motors (5 and $40 \mathrm{HP})$ and it is compared with the genetic algorithm and the classical parameter estimation method. Simulation results show that the proposed PSO method was indeed capable of estimating the parameters over a wide operating range of the motor.

\subsection{Multiobjective optimal design of three- phase induction Generator using simulated Annealing technique}

In this paper [19], the task of finding optimal design of a three-phase self-excited induction generator has been formulated as a multi criterion optimization problem. Criterial functions are the active material cost and capacitance required for excitation under full load conditions to maintain rated voltage. The problem is solved by giving weights which reflect the priority of objective functions. The Simulated Annealing technique is used as a tool to solve the problem. The proposed approach is implemented for the optimal design of a sample induction machine (15HP, $440 \mathrm{~V}, 6$ pole, $50 \mathrm{~Hz}$ ) operating as induction generator. The overall cost of an induction generator is reduced significantly when designed on the basis of minimizing both active material cost and capacitance required. The obtained results prove the effectiveness of a multi objective approach since it allows us to find a good compromise among the proposed goals, and above all it represents an efficacious tool for the designer. 


\subsection{Multiobjective design optimization of five-phase Halbach Array Permanent- Magnet machine}

In this paper [20], a five-phase Halbach array permanentmagnet machine was optimally designed for high dynamic performance such as high efficiency, high steady-state torque, a very high torque during transient period of time and low rotational inertia. A detailed and accurate analytical model of the machine considering the end winding effects was proposed to evaluate the torque, efficiency and rotational inertia of the motor. Some key parameters of the machine are chosen as the optimization variables to optimize the main machine design objectives. A Genetic Algorithm technique is used to optimize the objectives function. The machine is designed optimally for each object separately and then the whole objects are optimized simultaneously. The detailed finite-element analysis results are carried out to evaluate and verify the performance of the designed machine.

\subsection{Multiobjective shape optimization of segmented pole Permanent-Magnet Synchronous machines with improved torque characteristics}

A multiobjective optimization process has been proposed in this paper [21] is used to reduce the cogging torque with minimum loss in the output torque in PMSMs. A novel semianalytical model with improved accuracy and computational efficiency has been developed for cogging torque calculation in the segmented pole machines and used in the optimization process. The cogging torque calculation method first computes the air gap flux density distribution of the equivalent slot less segmented machines and effects of slots are taken into account by the air gap permeance function calculated by a static FEA. To achieve the best configuration i.e, to minimize the fitness function, particle swarm optimization has been employed. The proposed optimization framework has been applied to segmented pole machines with two (case 1) and three (case 2) segments per pole. In case 1, a cogging torque reduction by about $96 \%$ was obtained while the first harmonic of air gap flux density is reduced by $3.5 \%$ with respect to the initial non segmented machine with the same magnet volume. In case 2 , the cogging torque reduction is about $91 \%$ regarding the non segmented machine with the similar magnet volume while flux density loss is less than $0.5 \%$.

\subsection{A novel method for multiobjective design and optimization of three phase induction machines}

A fast and efficient multiobjective optimization design method is developed for induction machines, which requires much fewer design iterations than the traditional design method and only six independent design variables are needed to start the design has been proposed in this paper [22]. This paper also points out the complexities in design optimization caused by the limitation in selecting the number of turns and specific challenges in electrical machine design are emphasized. A canonical particle swarm optimization (PSO) method with penalty function for design constraints is developed to find the optimal solution for a user-defined objective function. A comparison study of PSO and genetic algorithm (GA) is also and the comparison shows that PSO is more successful in finding the global optima and also has better computational efficiency than GA. The original contributions of this paper are a novel induction machine design method, consideration of winding turn selection limitation and a machine-design-focused comparison.

\subsection{Evolutionary computation based multi-objective pole shape optimization of switched reluctance machine}

A multi-objective evolutionary algorithm approach for determination of optimum pole shape of SRM is proposed in this paper [23], considering average torque, torque ripple and copper loss as objectives. The application of elitist Nondominated Sorting Genetic Algorithm version II (NSGAII) to determine optimum pole shape design for performance enhancement of Switched Reluctance Machine (SRM) is presented. In SRM, torque output and torque ripple are sensitive to stator and rotor pole arcs and their selection is a vital part of SRM design process. The problem of determining optimal pole arc is formulated as a multiobjective optimization problem and the Finite Element Method (FEM) is used to determine the performance of the machine. NSGA-II is used in the search for Pareto solutions and the proposed optimization technique is applied to determine optimal pole shape of an 8/6, four-phase, $5 \mathrm{HP}$, $1500 \mathrm{rpm}$ SRM. The Pareto fronts obtained using the proposed approach is in close agreement with the fronts obtained using weighted sum method. The results indicate that the optimization algorithm has yielded new motor designs improving the three objectives considered. Analyzing the performance of the machine using FEA confirm the application of NSGA-II to determine various viable pole shape designs for performance enhancement of SRM. The results show the effectiveness of the proposed approach and confirm the application of NSGA-II as a promising tool for solving SRM design problems. The results obtained by NSGA-II are compared and validated with classical multiobjective approach based on weighted sum method using Differential Evolution (DE) algorithm.

\subsection{Multiobjective optimal design of low- speed linear induction motor using genetic algorithm}

In this paper [24], a computer aided systematic and applicable design algorithm has been proposed for single-sided linear induction motor (SLIM). In the proposed algorithm, different geometries of the machine are calculated using analytical equations. The low-speed SLIM design is optimized based on the equivalent circuit model and using Genetic algorithm. In order to maximize the efficiency and power factor as well as to minimize the primary weight, 9 effective variables are chosen for design optimizations which are effective in performance of the SLIM, are considered in optimization. 2D finite element method is employed to confirm the precision of the equivalent circuit model and the effectiveness of the optimization method. The FEM results which considers the end effects are in good agreement with the analytical results.

\section{CONCLUSION}

The state of the art study of MO used in electric machine optimization is first of all important to give an overview over what has been done. It also gives an overview on which area MO has been used and how successful the optimization was. Most of the authors conclude MO to be a promising optimization method, although there are some conclusions on premature convergence. Literature address the challenge connected to choice of selection of the optimization technique. There is however not a clear advice on this choice. 
Research in the field of electric machine design has also addressed this topic. With reference to the case study on Multiobjective Optimization in electrical Machines, it is found that design of electrical machines using conventional and evolutionary techniques gave the result to some extent. Instead, hybrid evolutionary techniques may be employed for the optimal design of machines.

\section{REFERENCES}

[1] Coello Coello C.A., Van Veldhuizen D.A., Lamont G.B., Evolutionary Algorithms for Solving Multiobjective Problems, Kluwer Academic Publishers, 2002.

[2] Deb.K, Multiobjective Optimization using Evolutionary Algorithms, John Wiley \& Sons Ltd., 2001.

[3] Ehrgott. M, Multicriteria optimization, Springer - Berlin, New York, 2000

[4] Miettinen K. M., Nonlinear Multiobjective Optimization, Kluwer Academic Publishers, 1999.

[5] Jahn. J, Vector Optimization: Theory, Applications, and Extensions, Springer Verlag, 2004.

[6] Osyczka. A, Multicriteria optimization for engineering design, edited by J.S. Gero, Design Optimization, Academic Press, 1985, pp. 193-227.

[7] Cohon. J.L and Marks. D.H., A Review and Evaluation of Multiobjective Programming, Water Resources Research, 11(2), 1975, pp. 208-220.

[8] Zadeh. L.A, Optimality and Non-scalar Valued Performance Criteria, IEEE Transactions in Automatic Control, AC-8(1), 1963, pp. 59-60.

[9] Marglin. S., Public Investment Criteria, MIT Press, Cambridge, Massachusetts, 1967.

[10] Stewart. T.J, Convergence and Validation of Interactive Methods in MCDM: Simulation Studies, edited by M.H. Karwan, J. Spronk and J. Wallenius, Essays in Decision Making: A Volume in Honor of Stanley Zionts, Springer-Verlag, 1997, pp. 7-18.

[11] Fogel, Evolutionary Computation: The Fossil Record. IEEE Press, 1988.

[12] Fogel, L. J., Owens, A. J., and Walsh, M. J., Artificial Intelligence through Simulated Evolution. John Wiley \& Sons, 1966.

[13] Lampinen. J, Differential Evolution - new naturally parallel approach for engineering design optimization, edited by B.H.V. Topping, Development in computational mechanics with high performance computing, Civil-Comp Press, Edinburgh, 1999, pp. 187197.

[14] Mimi Belatel, Hocine Benalla, "A Multiobjective Design Optimization of Induction Machine using CAD and
ANNs", ICGST-AIML Journal, ISSN: 1687-4846, Vol. 8, Issue II, September 2008, pp.1-8.

[15] Mehmet Cunkas, "Design Optimization of Electric Motors by Multiobjective Fuzzy Genetic Algorithms", Journal of Mathematical and Computational Applications, Vol. 13, No. 3, pp. 153-163, 2008.

[16] Yon-Do Chun, Pil-Wan Han, Jae-Hak Choi, Dae-Hyun Koo, "Multiobjective Optimization of Three-Phase Induction Motor Design Based on Genetic Algorithm", Proceedings of the 2008 International Conference on Electrical Machines, Paper ID 1220, pp.1-4

[17] Xue X. D, Cheng K.W. E, Ng T.W, and Cheung N. C, "Multi-Objective Optimization Design of In-Wheel Switched Reluctance Motors in Electric Vehicles", IEEE Transactions on Industrial Electronics, Vol. 57, No. 9, September 2010, pp.2980-2987.

[18] Sakthivel V.P, Bhuvaneswari. R, Subramanian. S, "Multi-objective parameter estimation of induction motor using particle swarm optimization", Engineering Applications of Artificial Intelligence, 2010, pp. 302312 .

[19] Kannan. R, Dr.Subramanian. S, Dr. Bhuvaneswari. R, "Multiobjective Optimal Design of Three-Phase Induction Generator using Simulated Annealing Technique", International Journal of Engineering Science and Technology, Vol. 2(5), 2010, pp. 1359-1369.

[20] Siavash Sadeghi and Leila Parsa, "Multiobjective Design Optimization of Five-Phase Halbach Array PermanentMagnet Machine", IEEE Transactions on Magnetics, Vol. 47, No. 6, June 2011, pp.1658-1666.

[21] Mahdi Ashabani and Yasser Abdel-Rady I. Mohamed, "Multiobjective Shape Optimization of Segmented Pole Permanent-Magnet Synchronous Machines with Improved Torque Characteristics", IEEE Transactions on Magnetics, Vol. 47, No. 4, April 2011, pp.795-804.

[22] Yao Duan and Ronald G. Harley, "A Novel Method for Multiobjective Design and Optimization of Three Phase Induction Machines", IEEE Transactions on Industry Applications, Vol. 47, No. 4, July/August 2011, pp.17071715 .

[23] Balaji. M, Kamaraj. V, "Evolutionary computation based multi-objective pole shape optimization of switched reluctance machine", Electrical Power and Energy Systems, 2012, pp. 63-69.

[24] Abbas Shiri, Abbas Shoulaie, "Multi-objective optimal design of low-speed linear induction motor using genetic algorithm", Electrical Review, ISSN 0033-2097, R. 88 NR 3b/2012. 